\title{
Editorial
}

\section{Nano/Microstructured Materials 2014}

\section{Amir Kajbafvala, ${ }^{1}$ Hamed Bahmanpour, ${ }^{2}$ Ali Moballegh, ${ }^{1}$ Mohammad H. Maneshian, ${ }^{3}$ and Hamid Reza Zargar ${ }^{4}$}

\author{
${ }^{1}$ Department of Materials Science and Engineering, North Carolina State University, 911 Partners Way, Raleigh, NC 27695-7907, USA \\ ${ }^{2}$ Department of Chemical Engineering and Materials Science, University of California, Davis, CA 95616, USA \\ ${ }^{3}$ Micron Technology Inc., Arlington, VA 22204, USA \\ ${ }^{4}$ Department of Metals and Materials Engineering, University of British Columbia, Vancouver, BC, Canada V6T $1 Z 4$
}

Correspondence should be addressed to Amir Kajbafvala; akajbaf@ncsu.edu

Received 8 February 2015; Accepted 8 February 2015

Copyright (C) 2015 Amir Kajbafvala et al. This is an open access article distributed under the Creative Commons Attribution License, which permits unrestricted use, distribution, and reproduction in any medium, provided the original work is properly cited.

Recently, there is a growing interest among materials scientists and chemists to generate a diverse range of organic and inorganic molecules and compounds using eco-friendly synthesis routes, including low-cost and short synthesis times. Therefore, a primary driver of synthetic chemistry is the development of efficient and environmentally benign synthetic protocols. Most recently, many works have been published on the synthesis and characterization of various multidimensional micro/nanostructured materials by use of different synthesis methods. However, complex conditions and long synthesis time were required for most of these technologies. The main purpose of this special issue was to investigate facile and rapid synthesis methods of organic and inorganic micro/nanostructured materials, in order to maximize the efficient use of safer and cheaper raw materials, and to reduce waste for fabrication of various micro/nanostructured materials.

Original review and research articles including experimental, theoretical, or simulations works were invited. Some of the potential proposed topics were rapid, low-cost, and eco-friendly synthesis routes of micro/nanostructured materials, utilizing advanced characterization techniques for studying nanostructured materials, microwave assisted synthesis methods, green synthesis methods for fabrication of zero-, one-, and two-dimensional nanomaterials, their benefits, and their capabilities for mass production. It is worth to mention that this special issue was first lunched in 2013 and received a high number of articles. Due to its initial success, it was launched again in 2014 and it has been selected as an annual issue for Journal of Nanoparticles. This 2014 annual issue contains six articles including one review and five research articles.

In their paper, "Role of Surfactant in the Formation of Gold Nanoparticles in Aqueous Medium," A. Das et al. stabilized L-tryptophan reduced gold nanoparticles in aqueous solution by use of sodium dodecyl sulphate (SDS). Their results revealed that SDS plays an important role in the formation mechanism of the gold nanoparticles. Moreover, the reaction kinetics of the gold nanoparticles is easily observable by a steady-state spectroscopic method such as UV-Vis spectroscopy unlike $\mathrm{NaBH}_{4}$ where the reaction is too fast and citrate method where heating is required to initiate the reaction. The kinetic details of the formation processes of gold nanoparticles reveal the bimodal mechanism. The observation of formation of gold nanoparticles of two different sizes, spaced over a time gap of about 30 minutes during the process of synthesis, opens up the prospect of utilizing the same reaction to tune nanoparticles size. The authors have summarized that this study guides attentions towards the potential usage of anionic surfactants in tuning the size of gold nanoparticles along with their stabilization.

In their research article "Biogenic Synthesis of Silver Nanoparticles Using Scenedesmus abundans and Evaluation of Their Antibacterial Activity," N. Aziz et al. synthesized silver nanoparticles (AgNPs) using the cell free extract of Scenedesmus abundans with $\mathrm{AgNO}_{3}$. They concluded that the synthesized AgNPs through in vitro bioreduction system from unicellular microalga Scenedesmus abundans produced 
significant amount of nanoparticles when it is exposed to $1 \mathrm{mM}$ silver nitrate solution. These nanoparticles show a broad spectrum of antimicrobial activity against both Grampositive and Gram-negative bacteria.

In the review article, "Synthesis of Silver Nanoparticles in Photosynthetic Plants," R. Prasad focused on the synthesis of nanoparticles with special emphasis on the use of plants parts for the synthesis process, its applications, and future prospectus. As one main conclusion, the author stated that the antimicrobial activity of the nanoparticles had a wide scope in the preparation of target based drug delivery and clinical diagnostics system. The identification of the specific mechanism of the AgNPs inhibition of the microbial growth or the lethal effect provides major application in the medicine, environment, soil fertility, and water quality. Moreover, the synthesis of nanoparticles with different sizes and shapes is the basic challenging task in the green synthesis of nanoparticles; this requires a basic understanding of the nuclei formation and the influence of reaction species in nuclei morphology, but still there is dormancy about the actual mechanism of the nanoparticles synthesis from the biological mode of synthesis.

In "Plasmonic and Thermooptical Properties of Spherical Metallic Nanoparticles for Their Thermoplasmonic and Photonic Applications," V. K. Pustovalov et al. investigated the influence of nanoparticles parameters (type of metal, radii, optical indexes, density, and heat capacity of nanoparticle material), characteristics of radiation (wavelength and pulse duration), and ambient parameters on plasmonic and thermophysical properties of nanoparticles. Based on this study, it was established that the maximum value of thermooptical parameter (maximum nanoparticle temperature) can be achieved with the use of absorption efficiency factor of nanoparticle which is smaller than its maximum value.

In their research article, "Structured $\mathrm{Pd} / \gamma-\mathrm{Al}_{2} \mathrm{O}_{3}$ Prepared by Washcoated Deposition on a Ceramic Honeycomb for Compressed Natural Gas Applications," M. Adamowska and P. Da Costa studied the preparation of a coating procedure, from the washcoating with $\gamma$-alumina to the deposition of palladium by excess solvent or incipient wetness impregnation. The powder and the washcoat layers were studied by different characterization techniques such as SEM, BET surface area, and XRD. Vibration-resistance and heatresistance were also evaluated. It was shown that the alumina layer is quite well deposited on the channel walls. However, a detachment of the washcoat layer was observed after ultrasound treatment. It was proved that the monolith $\mathrm{Pd}$ impregnation method by incipient wetness impregnation is more efficient. The presence of palladium was confirmed using HRTEM, the palladium was present under the Pd (II) oxidation state, and the size of PdO particles varies between 2.5 and $3.1 \mathrm{~nm}$. The catalytic properties of the monolith catalyst were carried out using temperature programmed surface reaction (TPSR).

In, "Modulatory Effect of Citrate Reduced Gold and Biosynthesized Silver Nanoparticles on $\alpha$-Amylase Activity," K. Saware et al. described a novel approach to study the interaction of amylase enzyme with gold nanoparticles (AuNPs) and silver nanoparticles (AgNPs) and checked its catalytic function. AuNPs were synthesized using citrate reduction method and AgNPs were synthesized using biological route employing Ficus benghalensis and Ficus religiosa leaf extract as a reducing and stabilizing agent to reduce silver nitrate to silver atoms. A modulatory effect of nanoparticles on amylase activity was observed. Gold nanoparticles are excellent biocompatible surfaces for the immobilization of enzymes. Immobilized amylase showed 1- to 2-fold increase of activity compared to free enzyme. The biocatalytic activity of amylase in the bioconjugate was marginally enhanced relative to the free enzyme in solution. The bioconjugate material also showed significantly enhanced $\mathrm{pH}$ and temperature stability. The results indicate that the present study paves way for the modulator degradation of starch by the enzyme with AuNPs and biogenic AgNPs, which is a promising application in the medical and food industry.

\section{Acknowledgments}

At the end, the guest editors would like to express sincere appreciation to all the authors who have contributed in this 2014 annual issue. Moreover, our thanks are also extended to all of the reviewers for their valuable time and for enhancing the quality of these articles.

\author{
Amir Kajbafvala \\ Hamed Bahmanpour \\ Ali Moballegh \\ Mohammad H. Maneshian \\ Hamid Reza Zargar
}



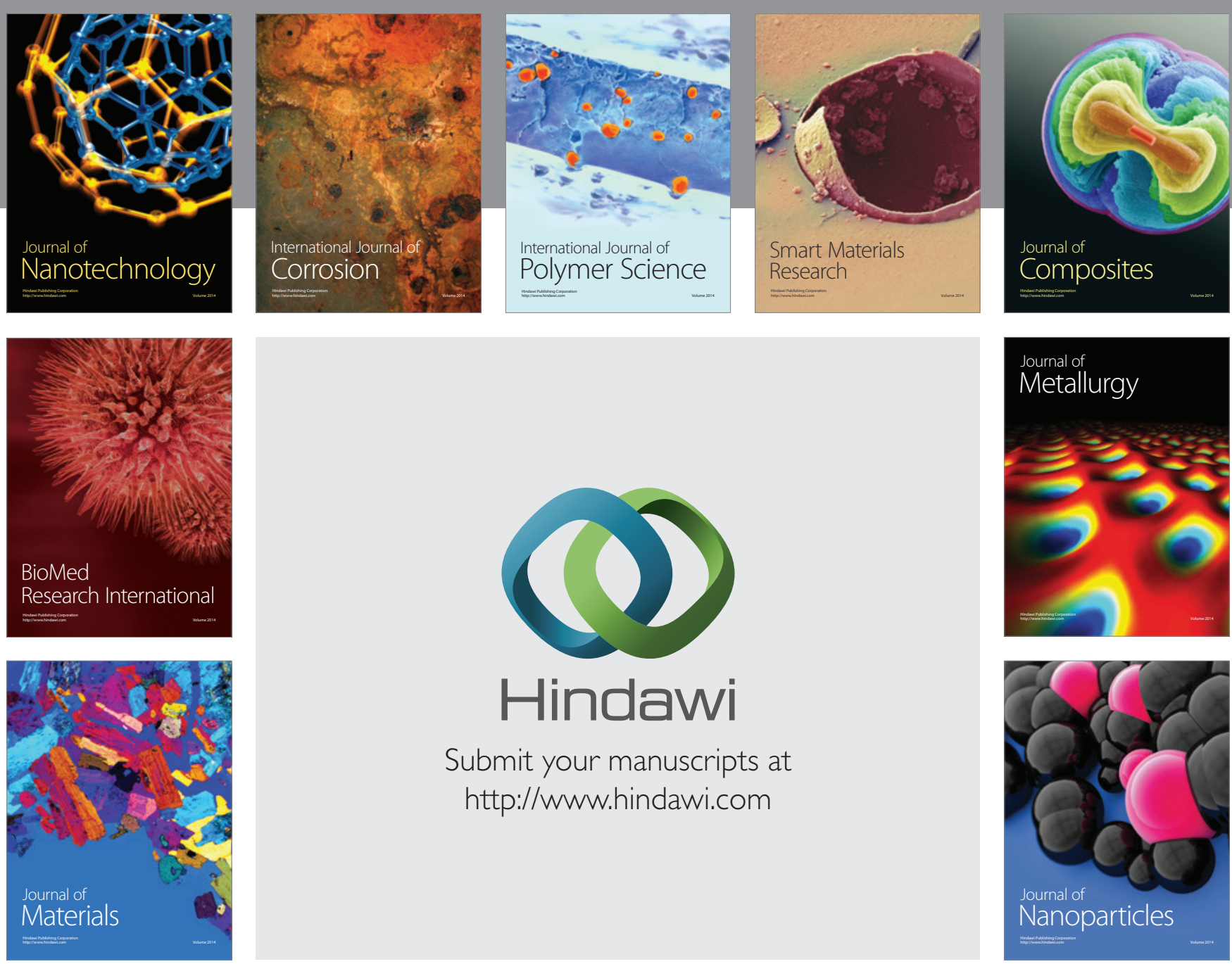

Submit your manuscripts at http://www.hindawi.com
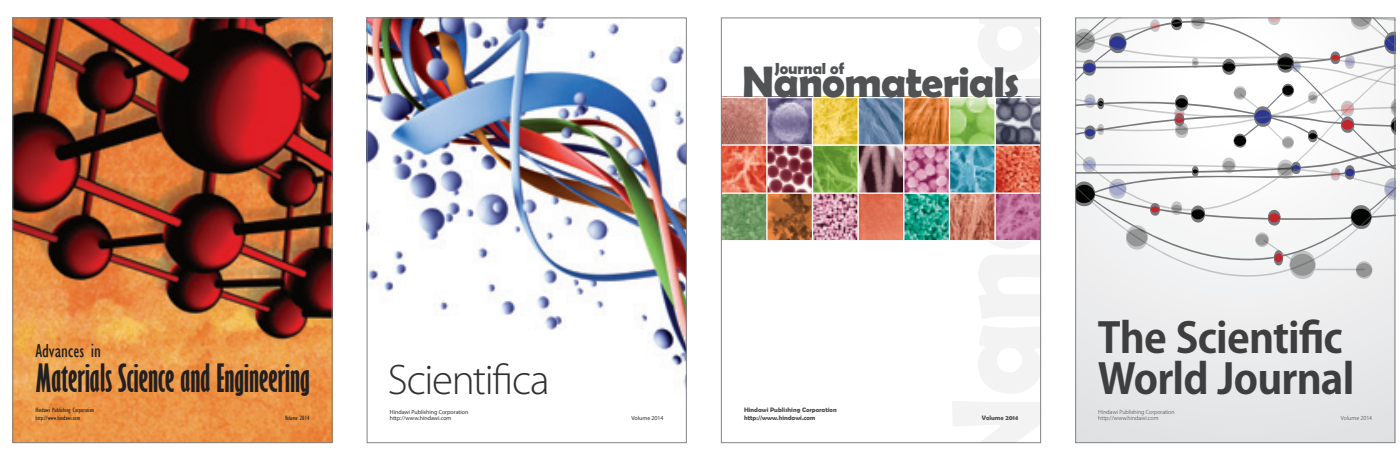

\section{The Scientific World Journal}
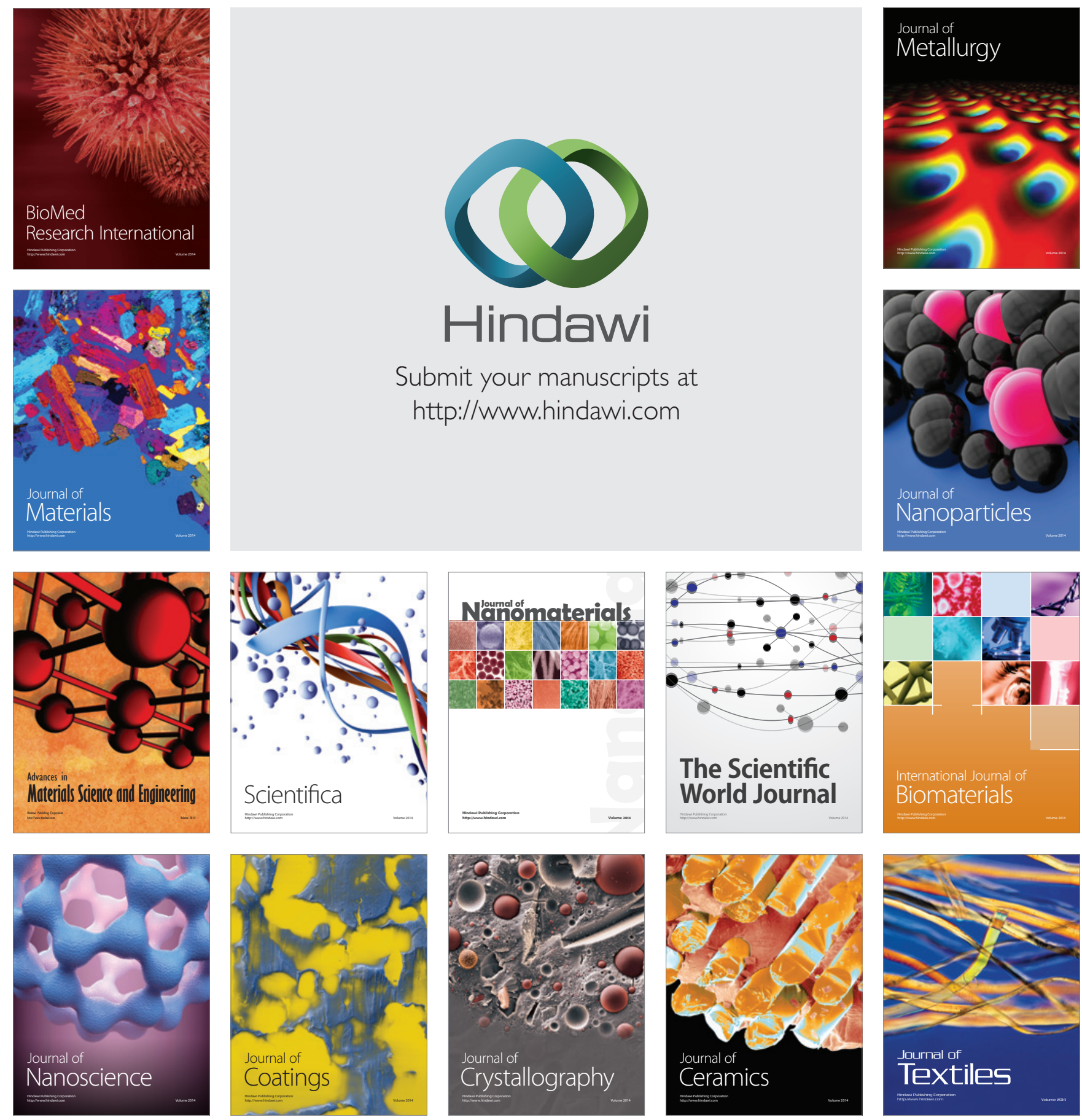